\title{
Editorial
}

\section{Digital coronary angiography: the new gold standard?}

Few, if any, other areas of imaging require the high spatial, temporal, and contrast resolutions that are needed for coronary arteriography. For two decades $35 \mathrm{~mm}$ cineangiography has fulfilled these requirements and has been regarded as the "gold standard" for the investigation of coronary artery disease; but despite its many advantages cine film has some drawbacks. Satisfactory film processing requires very careful attention to detail and is generally carried out after procedures are completed (a particular problem in some interventional procedures). Storage of film can occupy considerable space, contrast medium and radiation dosages are relatively high, and, above all, no quantitative analysis or image manipulation is possible. Digital angiography was being developed as early as $1975^{1}$ and is now well established in non-cardiac angiography. Cine angiography, however, remained unchallenged as the imaging technique of choice for coronary artery studies right through the early development period of digital angiography whose potential for coronary imaging has only been recognised within the past few years. ${ }^{23}$ Now that most major manufacturers of diagnostic $x$ ray equipment are offering commercially competitive digital cardiac imaging equipment the question increasingly being asked is "Has digital imaging become the new 'gold standard' for coronary angiography?”

\section{Overcoming limitations}

Early attempts at digital coronary angiography used systems developed for peripheral angiography. Although their performance was adequate for peripheral angiography, the speed of image acquisition (up to eight frames per second), matrix size (initially $256^{2}$ ), limited digital data storage (early digital discs were unable to hold even one patient's set of coronary angiograms), and the lack of adequate archiving made digital coronary angiography a cumbersome and inadequate examination. Imaging by continuous fluoroscopy with suboptimal radiographic variables produced very noisy images with much motion artefact and this severely restricted the capabilities of early systems. Technical developments occurred with increasing rapidity in the past decade and by 1985 digital cardiac imaging had progressed to a point where its potential was becoming clear.

Dedicated cardiac digital systems were found to be of particular use in the study of congenital heart disease $e^{4-6}$ where the demands for spatial resolution were not quite as high as for coronary studies. It was clear that the ability to manipulate digital data had major potential advantages, not only by increasing contrast sensitivity and permitting instant subtraction techniques but also by allowing much easier quantitative analysis and other manipulation of the angiographic data. It was also clear that for paediatric imaging digital angiography allowed satisfactory anatomical imaging with worthwhile reductions in contrast and radiation dose. ${ }^{78}$

\section{Early experience}

Early studies with digital subtraction imaging of intravenous left ventriculography (IV-DSA) and low volume intra-arterial ventriculography (IA-DSA) showed that it was possible to obtain satisfactory quality ventriculograms without the arrhythmias that often degrade conventional ventriculograms. Quantification of right and left ventricular function (by either edge detection methods or videodensitometry) was easier than with cine film. Regional wall motion analysis was achieved with better spatial resolution than with radionuclide ventriculography. Digital angiography made it possible to perform exercise ventriculography by either IV-DSA or low volume IA-DSA with better spatial resolution than with radionuclide ventriculography. ${ }^{910}$

\section{Current experience}

Recent developments in digital cardiac imaging have been dramatic. Pulsed imaging using more advantageous radiographic factors has led to greatly improved signal to noise ratios, elimination of motion artefact, easier mask mode subtraction (especially with ECG advancement of the mask), and has been accompanied by improvements in image intensifier performance and optimisation of the television chain for digital rather than analogue image acquisition. Pulsed acquisition improves the signal to noise ratio by reducing the quantum mottle, which is particularly obvious with the high contrast resolution of digital systems. The intrinsic spatial resolution of a new caesium iodide image intensifier is typically $4-5$ line pairs $/ \mathrm{mm}$ but this can deteriorate to as low as 2 line pairs/mm over time. This compares with a resolution of about 2 line pairs $/ \mathrm{mm}$ for a $512^{2}$ matrix on a $17 \mathrm{~cm}$ image intensifier. Although this spatial resolution may be inferior to that of cine film, the greater contrast sensitivity compensates for the poorer spatial resolution so that the overall perceived resolution, which contains the diagnostic information, is similar or better than for conventional cine studies. Commercially available cardiac digital systems commonly offer acquisition on a $512^{2}$ matrix at 25 frames per second. $1024^{2}$ matrices are now available, although with lower frame rates. Although the denser matrix has a resolution approaching that of the best image intensifiers it is not clear whether this produces more diagnostic information. The use of a $1024^{2}$ matrix does require the use of a higher radiation dose to reduce the effect of quantum mottle and produces four times more data for storage than a $512^{2}$ matrix, typically limiting image acquisition to 6 frames per second. Biplane studies can be recorded on digital imaging but if the computer shares its function between two planes, the frame rate of each will be halved. It has now been shown in several studies that digital coronary imaging compares very favourably with cine imaging in fulfilling the exacting demands of coronary arteriography. ${ }^{1112}$

Improvements in computer design allow the acquisition 
and processing of huge quantities of data. Less than 10 years ago the best systems could only acquire data at 8 frames per second $\left(512^{2}\right.$ matrix) with a maximum of 300 images being stored on the hard disc ( 37 seconds or about half of one complete examination). Image analysis and selection for archiving (to analogue video tape or sheet film) had to be done during the examination and had to be completed before the next patient could be examined. Simultaneous image acquisition and analysis was not possible. By using multiple hard discs, data compression techniques, fast processors, and task sharing a whole day's work can now be stored on the computer and can be analysed without disrupting subsequent examinations. Currently available equipment can store up to 26000 images. If single plane acquisition is on a $512^{2}$ matrix at 25 frames per second this allows the storage of about 17 minutes of filming (equivalent to 13 patients examined in 10 projections and imaged for an average of eight seconds in each projection).

\section{Advantages}

The obvious immediate advantage of high quality digital imaging is the availability of instant final quality images. This has particular advantages in monitoring the progress of coronary angioplasty or other interventional procedures $^{13}$ and various image storage and "road mapping" techniques have been used. Once the digital image has been acquired the data can be processed in various ways. Experience with current systems suggests that subtraction techniques add little to the usefulness of a digital coronary study. Changing contrast levels, windowing, and defining edges can improve the quality of the image and to some extent will compensate for any radiographic shortcomings. Automatic techniques for stenosis measurement or flow estimation can use edge defining techniques ${ }^{14}{ }^{15}$ or densitometry. ${ }^{16-18}$ Functional data related to regional blood flow can be derived by various analytical techniques. The precision with which digital data are presented should not obscure the fact that the technique has some inherent potential for inaccuracy, ${ }^{12}$ for example, flare, beam-hardening, and scatter may contribute to measurement errors which can in turn lead to errors in stenosis estimation.

Archiving and review of cine angiocardiograms has always been a significant problem with cine films requiring large amounts of storage space and dedicated projectors for viewing. Digital imaging is not without its storage and display problems also. Early digital images could only be archived as processed images on sheet film or on analogue videotape because of restrictions on the amount of digital data that could be stored on conventional floppy or hard discs. Much of the data acquired at great expense by using digital angiography was therefore lost. Archiving on analogue video cassette allows easy access to the images, provided that a separate cassette is used for each patient and high quality video display equipment is available; but downloading on to analogue videotape prohibits further digital image manipulation.

The development of digital video cassettes means that a complete digital examination can be archived and recalled at a later date for reanalysis. This can be an expensive and bulky option-though it still requires less storage space than cine film. Replay has to be through the digital processor to which access may be limited because the system may well be in use for examining another patient. Alternatively, the fully processed digital images can be recorded on to high resolution mini-video cassettes, which have a much better performance than conventional video cassettes, take up little storage room, and can be replayed through conventional video recorders. Quantitative data on ventricular function and stenosis estimation can be recorded on a video cassette or hard copy imager such as a laser imager. At present there is still no generally available, cheap, and convenient method of recording and displaying the digital data for routine clinical viewing, but newer forms of data storage will become available to overcome these problems. The decisions in selecting a new digital system will be many, but one of the most important will be the mode of image storage and the display that best suits the working patterns of individual departments.

Radiation dosage for both patient and staff can be reduced by the use of digital imaging but such reductions may not be substantial if image quality is to be maintained. High quality images still require high doses of radiation to produce sufficient image detail (reduce quantum mottle) and it is not primarily in the radiation dose per frame that reductions are easiest to achieve, though some reduction is possible. Important radiation dose reduction in coronary arteriography has been shown in some systems by the use of "gap-filling" techniques in which the $x$ ray pulse rate can be halved (for example from 25 to $12 \frac{1}{2}$ frames per second) but each frame is digitally duplicated. This eliminates flicker but still allows high quality diagnostic images to be presented smoothly to the viewer. This technique halves radiation doses. In an analogous fashion, digital pulsed fluoroscopy systems can also reduce radiation doses. The capital cost of a new digital system is a little greater than for a conventional system but the difference is getting smaller. There are savings to be made with digital systems, particularly in running costs. Expensive cine film and processing equipment is eliminated as well as the staff time taken to do this. Contrast medium volumes or concentrations can be reduced too. Finally the speed of procedures can be increased because instant high quality images are available for review; this is particularly relevant to interventional work.

\section{Conclusion}

For a long time there has been doubt about whether the practical problems of digital angiocardiography, particularly those related to resolution and to image storage and review, have been overcome sufficiently to make it worth taking the major step from a cine to a digital department. With the currently available digital equipment which can acquire images of equal or better quality than cine film and store them with increasing ease, it seems that the case for going digital is now overwhelming and this will soon be the "gold standard" technique for coronary arteriography. ${ }^{19} 20$

Department of Clinical Radiology,

GEORGE HARTNELL

New England Deaconess Hospital,

Boston, Massachusetts 02215,

USA

Department of Radiodiagnosis,

Bristol Royal Infirmary,

Bristol BS2 $8 H W$

PETER WILDE

1 Heintzen PH, Brennecke R, Bursch JH. Automated videoangiographic image analysis. Computers in cardiology. Long Beach: IEEE Computer Society, 1975:55-64.

2 Mancini GBJ Higgins CB. Digital subtraction angiography; a review of Mancini GBJ, Higgins CB. Digital subtraction angiography

cardiac applications. Prog Cardiovasc Dis 1985;18:111-41. JJ Jr. Digital Neeley JP III, Vannier MW, Gutierrez
subtraction angiography of the coronary arteries. Crit Rev Diagn Imaging subtraction angiog $1985 ; 25: 23-60$.

4 Buonocore E, Pavlicek W, Modic MT, Meaney TF, O'Donovan PB, Grossman LB, et al. Anatomic and functional imaging of congenital heart Grossman LB, et al. Anatomic and functional imaging of congenital heart
disease with digital subtraction angiography. Radiology 1983;147:647-54. disease with digital subtraction angiography. Radiology $1983 ; 147: 647-54$.
5 Dickinson DF, Wilson N, Partridge JB. Digital subtraction angiography in infants and children with congenital heart disease. Br Heart $J$ 1984;

51:485-91.
6 Tonkin 'ILD, Fitch SJ, Magill HL, Gerald BE, Gold RE, Alpert BS, et al. Pediatric digital subtraction angiography: intra-arterial and intracardiac 
applications. Pediatr Radiol 1986;16:126-30.

7 Levin AR, Goldberg HL, Borer JS, Rothenberg LN, Nolan FA, Engle MA et al. Digital angiography in the paediatric patient with congenital heart
disease; comparison with standard methods. Circulation 1983;68:374-84.

8 Bogren HG, Bursch JH. Digital angiography in the diagnosis of congenital heart disease. Cardiovasc Intervent Radiol 1984;7:180-8.

9 Bellamy. GR, Yiannikas J, Detrano R, Simpfendorfer C, Salcedo EE. Detection of multivessel disease after myocardial infarction using intravenous stress digital subtraction angiography. Radiology 1986 161:685-9.

10 Detrano R, Yiannikas J, Simpfendorfer C, Hobbs RE, Salcedo EE. Exercise digital subtraction ventriculography for the detection of ischaemic wal motion abnormalities in patients without myocardial infarction. Br Heart $J$ 1986;56:131-7.

11 Goldberg HL, Moses JW, Fisher J, Tamari I, Borer JS. Diagnostic accuracy of coronary angiography utilizing computer-based digital subtraction methods: comparison to conventional cineangiography. Chest 1986;90: 793-7.

12 Gurley JC, Nissen SE, Booth DC, Harrison M, Grayburn P, Elion J, et al. Comparison of simultaneously performed digital and film-based angiography in assessment of coronary artery disease. Circulation 1988;78: $1411-20$.
13 Katritsis D, Lythall DA, Anderson MH, Cooper IC, Webb-Peploe MM. Assessment of coronary angioplasty by an automated digital angiographic

14 Ratib OM, Mankovich NJ. Quantitative coronary arteriography: design and validation. Radiology 1988;167:743-7.

15 Popma JJ, Eichhorn EJ, Dehmer GJ. In vivo assessment of a digital angiographic method to measure absolute coronary artery diameter. Am J Cardiol 1989;64:131-8.

16 Ikeda $H$, Koga Y, Utsu F, Toshima $H$. Quantitative evaluation of regional myocardial blood flow by videodensitometric analysis of digital subtraction coronary arteriography in humans. J Am Coll Cardiol 1986;8:809-16.

17 Lefree MT, Simon SB, Mancini J, Bates ER, Vogel RA. A comparison of $35 \mathrm{~mm}$ cine film and digital radiographic image recording: implications for quantitative coronary arteriography. Invest Radiol 1988;23:176-83.

18 Simons MA, Muskett AD, Kruger RA, Klausner SC, Burton NA, Nelson JA. Quantitative digital subtraction coronary angiography using videodensitometry: an in vivo analysis. Invest Radiol 1988;23:98-106.

19 Pepine CJ. The cardiac catheterization laboratory-1990. Am J Cardiol 1990;66:37F-40F

20 Tobis J, Henry W, Nalcioglu O. Cardiac digital angiography. In: Brundage BH, ed. Comparative cardiac imaging. Rockville: Aspen, 1990:47-63.

\section{PLANTS IN CARDIOLOGY}

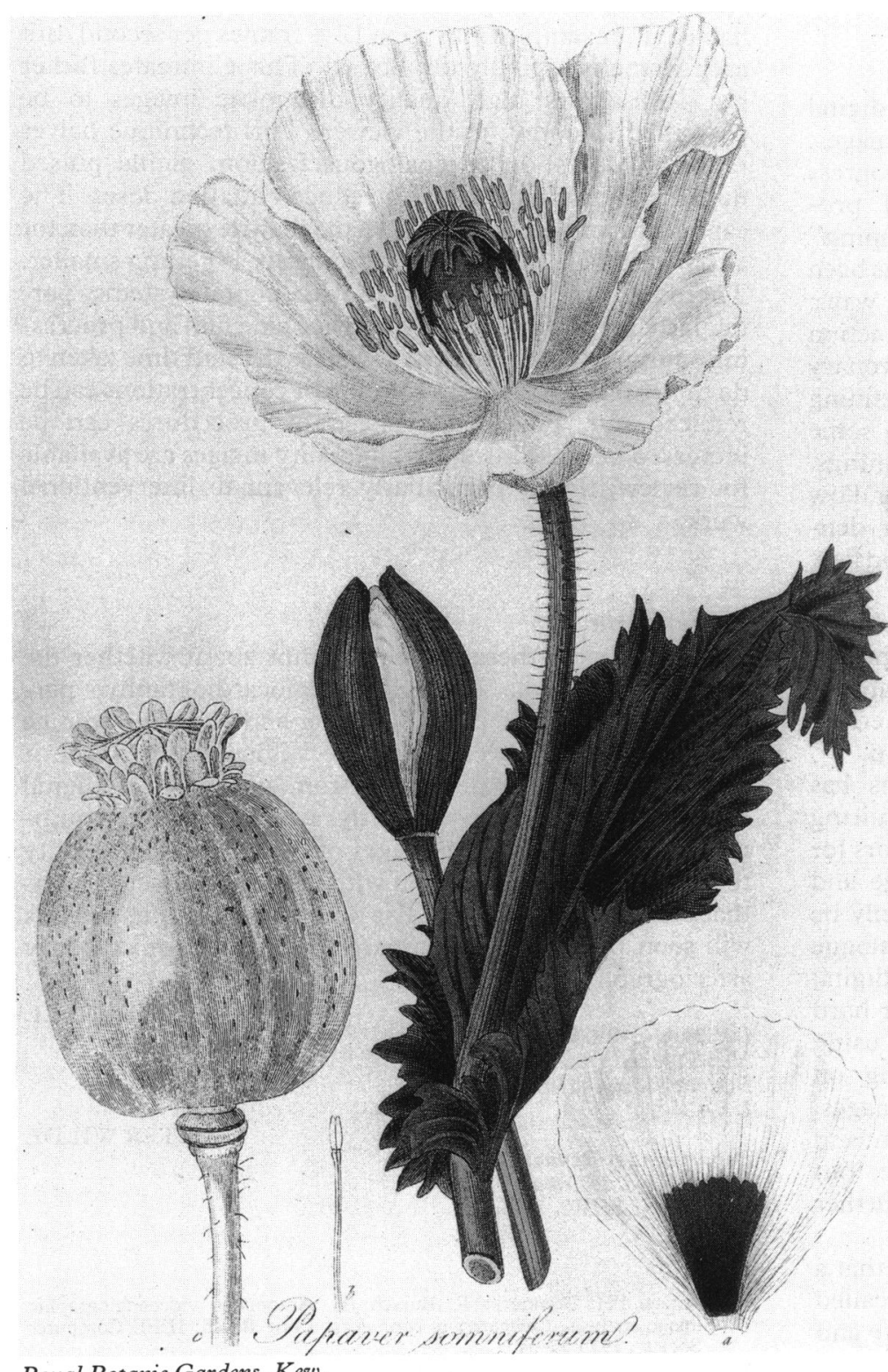

Royal Botanic Gardens, Kew.

Papaver somniferum $\mathrm{L}$. Stephenson J, Churchill J M. Medical botany. London: 1836, volume 3, plate 159.
When the unripe seed capsule of the opium poppy Papaver somniferum (Papaveraceae) is incised a milky fluid exudes. The dried juice, opium (from the Greek, opos, juice), has been used medicinally for over 5000 years. The 25 or so alkaloids of opium belong to two distinct chemical classes with quite different actions. Morphine and codeine belong to the phenanthrene class. Papaverine, which accounts for only $1 \%$ of the alkaloids, is in the benzylisoquinoline class and was isolated in 1848. But its lack of analgesic activity inhibited pharmacological investigation until 1917 when David Macht initiated this at Johns Hopkins Medical School. Because it relaxes smooth muscle it is a good vasodilator; in cardiac muscle it depresses conduction and prevents chloroform induced ventricular fibrillation. But PaulD White found it to be of little use in angina or hypertension and it was tried without success in cardiac arrhythmias. Nevertheless, papaverine and its synthetic analogues were popular as antispasmodic drugs for gastrointestinal and genitourinary ailments and in 1937 the German pharmaceutical firm Knoll asked their chemist, Ferdinand Dengel, to synthesise it. He worked on the compound for over 20 years and in May 1959 he produced an analogue, D365 (D for Dengel), which was soon shown to be pharmacologically much more active than other analogues or other similar drugs. Unlike other vasodilators it had negative inotropic and chronotropic effects. It was iproveratril, later to be called verapamil, and was marketed as Isoptin. Because it was thought to be a $\beta$ blocker clinical trials in angina began in 1961 . Fleckenstein's study of verapamil started in 1963 and led to his seminal discovery of calcium antagonism as its mode of action. In 1972 Schamroth, Krikler, and Garrett (British Medical Journal 1972;i:660-2) were the first to link the clinical action of verapamil in terminating arrhythmias with Fleckenstein's concept of calcium channel blockade.

Morphine, still pre-eminent for pain relief, was formerly valuable in acute left ventricular failure, and in 1942 Crighton Bramwell and J T King said, "morphine acts as a specific and what is more it is the only drug which is effective."

Papaver somniferum originated in the western Mediterranean and is cultivated chiefly in Asia and Tasmania. Its seed is free of opium and is used on bread. The poppy family, Papaveraceae, has 23 genera and 210 species, mostly in the northern hemisphere. None of its other species has alkaloids that are either better or different from those in the opium poppy.

A HOLLMAN 